\title{
Interfacial waves due to a singularity in a system of two semi-infinite fluids
}

\author{
D. Q. $\mathrm{Lu}^{\mathrm{a})}$ \\ Shanghai Institute of Applied Mathematics and Mechanics, Shanghai University, Yanchang Road, \\ Shanghai 200072, China
}

Allen T. Chwang ${ }^{\text {b) }}$

Department of Mechanical Engineering, The University of Hong Kong, Pokfulam Road, Hong Kong, China

(Received 13 October 2004; accepted 16 September 2005; published online 28 October 2005)

\begin{abstract}
The three-dimensional interfacial waves due to a fundamental singularity steadily moving in a system of two semi-infinite immiscible fluids of different densities are investigated analytically. The two fluids are assumed to be incompressible and homogenous. There are three systems to be considered: one with two inviscid fluids, one with an upper viscous and a lower inviscid fluid, and one with an upper inviscid and a lower viscous fluid. The Laplace equation is taken as the governing equation for inviscid flows while the steady Oseen equations are taken for viscous flows. The kinematic and dynamic conditions on the interface are linearized for small-amplitude waves. The singularity immersed above or beneath the interface is modeled as a simple source in the inviscid fluid while as an Oseenlet in the viscous fluid. Based on the integral solutions for the interfacial waves, the asymptotic representations of wave profiles in the far field are explicitly derived by means of Lighthill's two-stage scheme. An analytical solution is presented for the density ratio at which the maximum wave amplitude occurs. The effects of density ratio, immersion depth, and viscosity on wave patterns are analytically expressed. It is found that the wavelength of interfacial waves is elongated in comparison with that of free-surface waves in a single fluid. (C) 2005 American Institute of Physics. [DOI: 10.1063/1.2120447]
\end{abstract}

\section{INTRODUCTION}

When a body moves with a constant velocity on or beneath the free surface of water, the well-known Kelvin ship waves are generated within a V-shaped region behind the moving body. In classical theories of ship waves, the water is regarded as an incompressible inviscid fluid, and the airwater interface is treated as a free surface with a constant atmospheric pressure. ${ }^{1,2}$ To remove the singular behavior predicted by the potential theory for the diverging waves near the moving path of a surface-floating pressure point, Cumberbatch $^{3}$ suggested an Oseen approximation for the ship waves due to a surface-piercing point pressure. To study the effect of viscosity on the free-surface waves due to a submerged body, Luyre ${ }^{4}$ proposed a theoretical formulation for the laminar interaction of viscous wakes with a free surface, in which the moving body is mathematically modeled as an Oseenlet, that is, the fundamental singularity of the steady Oseen flow. Furthermore, using Lighthill's two-stage scheme, ${ }^{5,6}$ which in essence involves the Cauchy residue theorem and the method of stationary phase, Luyre, ${ }^{7}$ Chan and Chwang, ${ }^{8}$ and $\mathrm{Lu}^{9}$ derived the asymptotic expansions of wave profiles in the far wake in a viscous fluid of infinite depth. Chan and Chwang ${ }^{10}$ also provided an integral solution for the image system of an Oseenlet bounded by a no-slip wall. Liu and Tao ${ }^{11}$ studied the viscous free-surface waves

\footnotetext{
${ }^{a)}$ Electronic mail: dqlu@mail.shu.edu.cn

b) Author to whom correspondence should be addressed. Telephone: (+852)

2859 2634. Fax: $(+852) 2858$ 5415. Electronic mail: atchwang@hkucc.hku.hk
}

due to a suddenly starting Oseenlet and found a finite region of validity for the steady-state solution. Lu and Chwang ${ }^{12}$ obtained the transient components of free-surface waves due to the impulsive motion of an Oseenlet. Taking the Oseen approximation, Liu and $\mathrm{Tao}^{13}$ and $\mathrm{Lu}^{14}$ analytically studied the free-surface waves and far wakes generated by a normal pressure point moving at the surface of a viscous fluid of infinite depth. Recently, the investigation on the ship wakes has increased largely because of the development of synthetic aperture radar. ${ }^{15,16}$

All the aforementioned works are based on a model which involves singularities moving in a single fluid with a free surface. To study the effect of air above the free surface on water waves, He et al. ${ }^{17}$ considered a simple, but analytically solvable model which involves a simple source moving in two semi-infinite inviscid fluids of different densities. Applying the method developed by Wehausen and Laitone, ${ }^{1} \mathrm{He}$ et $a{ }^{17}{ }^{17}$ derived the formal analytical solutions for the velocity potentials in terms of unevaluated double Fourier integrals only, from which the corresponding physical interpretation was not provided explicitly at that time. As a matter of fact, the motion of a body near the interface between two fluids is of fundamental interest due to its importance in many engineering applications. ${ }^{18}$ Based on the potential theory, Yeung and Nguyen ${ }^{19}$ and Gang et al. ${ }^{20}$ studied the waves due to a moving source and dipole in a two-layer ocean of finite depth, respectively. The preliminary mathematical formulation for the body-induced interfacial waves between two incompressible and homogenous fluids, inviscid or viscous, was provided by $\mathrm{Lu}$ and Chwang, ${ }^{21}$ in which the 
body is modeled as a potential doublet in the inviscid fluid and as an Oseen doublet in the viscous fluid. According to the basic idea of the singularity method, ${ }^{22}$ the effect of body can be simulated theoretically by a discrete or continuous distribution of various singularities in linearized flows. Therefore, the investigation on the flows due to a fundamental singularity is of principal interest since all higher-order singularities, such as doublet, can readily be derived from the fundamental one.

The objective of the present work is to analytically study the interfacial waves due to a simple source or an Oseenlet steadily moving above or beneath the interface of two semiinfinite immiscible fluids of different densities. A system with two inviscid fluids, a system with an upper viscous and a lower inviscid fluid, and a system with an upper inviscid and a lower viscous fluid are considered in Secs. II-IV, respectively. It is assumed that the density of the upper fluid is less than that of the lower one. The Laplace equation is taken as the governing equation for inviscid flows while the steady Oseen equations are taken for viscous flows. The kinematic and dynamic conditions on the interface are linearized for small-amplitude waves. The integral-form solutions for interfacial waves due to point singularities are formally derived for six cases. The corresponding asymptotic representations of wave profiles in the far field are derived by applying Lighthill's two-stage scheme. ${ }^{5,6}$ Discussion and conclusions are made in Secs. V and VI, respectively.

\section{INTERFACIAL WAVES BETWEEN TWO INVISCID FLUIDS}

\section{A. Mathematical formulation}

In this section, the two fluids are assumed to be inviscid, incompressible, and unbounded. As is well known, the fundamental singularity for an inviscid flow is a simple source, which represents a point mass source in the fluid. Cartesian coordinates are taken fixed on the singularity. The $x$ axis is along the straight path of the moving simple source while the $z$ axis points vertically upward. Thus the fluid is moving at a uniform velocity $U \mathbf{e}_{x}$, where $U$ is a constant and $\mathbf{e}_{x}$ is the unit vector in the $x$ direction.

For simplicity, we take $U$ as the characteristic velocity, $\rho_{2} U^{2}$ as a reference pressure, and the deep-water ship wavelength $U^{2} / g$ as a characteristic length, where $\rho_{2}$ is the density of the lower fluid and $g$ is the gravitational acceleration. Therefore, the dimensionless governing equations are

$$
\nabla^{2} \Phi_{m n}=\Delta_{m n} M \delta\left(\mathbf{x}-\mathbf{x}_{0}\right) \quad(m, n=1,2),
$$

where subscript $m$ stands for the fluid (1: upper; 2 : lower) containing an observation point $\mathbf{x}=(x, y, z)$ while subscript $n$ stands for the fluid containing a source point $\mathbf{x}_{0}=\left(0,0, z_{0}\right)$. $\Phi_{m n}$ is the velocity potential for the perturbed flow in fluid $m$ due to the singularity immersed in fluid $n . \Delta_{m n}$ is the wellknown Kronecker delta, $\delta()$ the Dirac delta function, $M$ the magnitude of the simple source. It should be noted that for $n=1, z_{0}>0$ while for $n=2, z_{0}<0$.

It is assumed that the wave amplitude is very small in comparison with the wavelength. Thus, the linearized bound- ary conditions will be applied on the undisturbed interface $(z=0)$. The kinematic conditions on the interface are given by

$$
\begin{aligned}
& \frac{\partial \Phi_{1 n}}{\partial z}=\frac{\partial \Phi_{2 n}}{\partial z}, \\
& \frac{\partial \xi_{n}}{\partial x}=\frac{\partial \Phi_{1 n}}{\partial z},
\end{aligned}
$$

where $\xi_{n}$ is the dimensionless elevation of the interface due to the singularity immersed in fluid $n$. Equation (2) represents the continuity of the normal velocity. Equation (3) implies that fluid particles once on the interface will always remain there. The dynamic condition on the interface is given by

$$
\sigma\left(\frac{\partial \Phi_{1 n}}{\partial x}+\xi_{n}\right)=\frac{\partial \Phi_{2 n}}{\partial x}+\xi_{n}
$$

where $\sigma=\rho_{1} / \rho_{2}, \rho_{1}$ is the uniform density of the upper fluid. Equation (4) represents the balance of the normal pressure on the interface. Since the finite disturbance caused by the moving source must die out at infinity,

$$
\nabla \Phi_{1 n} \rightarrow 0 \quad \text { as } z \rightarrow+\infty, \quad \nabla \Phi_{2 n} \rightarrow 0 \quad \text { as } z \rightarrow-\infty,
$$

which imposes a uniqueness on the problem concerned. Thus, the governing equation (1) and boundary conditions (2)-(5) constitute a well-posed boundary-value problem for $\Phi_{m n}$ and $\xi_{n}(m, n=1,2)$.

\section{B. Integral solutions}

The assumption of linearity allows us to envisage the perturbed flow as the sum of a singular and a regular flow. The former represents the effect of a moving singularity while the latter represents the influence of the interface. Thus, we write

$$
\Phi_{m n}=\Delta_{m n} \Phi_{m n}^{S}+\Phi_{m n}^{R},
$$

where $\Phi_{m n}^{S},(m, n=1,2)$ are the potentials due to the singularity while $\Phi_{m n}^{R},(m, n=1,2)$ are harmonic functions everywhere in the corresponding domain. The singular component is the fundamental solution of Laplace equation

$$
\Phi_{n n}^{S}=-\frac{M}{4 \pi r} \quad(n=1,2),
$$

where $r=\sqrt{x^{2}+y^{2}+\left(z-z_{0}\right)^{2}}$. Furthermore, by invoking Weyl's identity, ${ }^{23}$ Eq. (7) can be expressed by

$$
\Phi_{n n}^{S}=-\frac{M}{8 \pi^{2}} \int_{-\infty}^{+\infty} \int_{-\infty}^{+\infty} \frac{1}{K} \exp \left(i f-K\left|z-z_{0}\right|\right) d \alpha d \beta,
$$

where $K=\sqrt{\alpha^{2}+\beta^{2}}$ and $f=\alpha x+\beta y$. For the regular component in Eq. (6), we have

$$
\nabla^{2} \Phi_{m n}^{R}=0 .
$$

Thus, the relation between the singular and regular component can be established through the boundary conditions at the interface $(z=0)$, 


$$
\begin{aligned}
& \frac{\partial \Phi_{1 n}^{R}}{\partial z}-\frac{\partial \Phi_{2 n}^{R}}{\partial z}=-\Delta_{1 n} \frac{\partial \Phi_{11}^{S}}{\partial z}+\Delta_{2 n} \frac{\partial \Phi_{22}^{S}}{\partial z}, \\
& \frac{\partial \xi_{n}}{\partial x}-\frac{\partial \Phi_{1 n}^{R}}{\partial z}=\Delta_{1 n} \frac{\partial \Phi_{11}^{S}}{\partial z}, \\
& (\sigma-1) \xi_{n}+\sigma \frac{\partial \Phi_{1 n}^{R}}{\partial x}-\frac{\partial \Phi_{2 n}^{R}}{\partial x}=\Delta_{2 n} \frac{\partial \Phi_{22}^{S}}{\partial z} .
\end{aligned}
$$

Next we denote the Fourier transform with respect to the $x$ and $y$ of function $F(x, y, z)$ by $\widetilde{F}(\alpha, \beta, z)$. Taking Eq. (5) into consideration and applying the Fourier transform to Eq. (9), we obtain

$$
\Phi_{m n}^{R}=\frac{1}{4 \pi^{2}} \int_{-\infty}^{+\infty} \int_{-\infty}^{+\infty} \widetilde{\Phi}_{m n}^{R}(\alpha, \beta) \exp \left[i f+(-1)^{m} K z\right] d \alpha d \beta,
$$

where $\widetilde{\Phi}_{m n}^{R}(\alpha, \beta),(m, n=1,2)$, are unknown functions. By substituting Eqs. (8) and (13) into the Fourier transforms of boundary conditions (10)-(12), a system of simultaneous algebraic equations is set up for the unknown functions $\widetilde{\Phi}_{m n}^{R}(\alpha, \beta)$ and $\widetilde{\xi}_{n}(\alpha, \beta)(m, n=1,2)$, which can readily be solved. Upon some mathematical manipulations, the formal integral expression for the interfacial wave profiles can be written as

$$
\xi_{n}=\frac{\lambda}{4 \pi^{2}} \int_{-\infty}^{+\infty} \int_{-\infty}^{+\infty} \frac{A_{0 n}}{D_{0}} \exp (i f) d \alpha d \beta \quad(n=1,2),
$$

where

$$
\begin{aligned}
& A_{0 n}=(-1)^{n} i \sigma^{2-n} M \alpha \exp \left[(-1)^{n} K z_{0}\right], \\
& D_{0}=\gamma K-\alpha^{2},
\end{aligned}
$$

and $\quad \gamma=(1-\sigma) /(1+\sigma)=\left(\rho_{2}-\rho_{1}\right) /\left(\rho_{2}+\rho_{1}\right), \quad \lambda=1 /(1+\sigma)$ $=\rho_{2} /\left(\rho_{2}+\rho_{1}\right) . \gamma$ is the Atwood number. $D_{0}$ in Eq. (16) may be regarded as the dispersion function that defines the dispersion relation for the interfacial waves in the two superposed inviscid fluids of infinite depth.

\section{Asymptotic representations}

The integral in Eq. (14) represents the exact solution for the interfacial wave elevation due to the fundamental singularity immersed in the upper $(n=1)$ or lower $(n=2)$ fluid. The physical characteristics of the wave motion, however, are not explicitly seen in these integral solutions. As stated by Noblesse and Chen ${ }^{24}$ the Fourier integral representations of wave profiles can formally be decomposed into a near-field nonoscillatory component and a far-field wave component, and the near-field component vanishes rapidly as the distance from the singularity increases. Therefore, the far-field behavior of interfacial waves are of principal physical interest here. Next, Lighthill's two-stage scheme, ${ }^{5,6}$ which in essence involves calculating the $\alpha$ integration by the residue theorem and the $\beta$ integration by the method of stationary phase, will be employed to derive the asymptotic representation of inter- facial waves in the far field [see Appendix]. Finally, we have the asymptotic representations for the far-field interfacial waves as

$$
\begin{gathered}
\xi_{n}=(-1)^{n} \sigma^{2-n} \lambda \gamma^{1 / 2} M d^{R} \sum_{k=1}^{2} d_{k}^{S} Q_{k}^{3 / 4} \cos \varphi_{k}+o\left(\frac{1}{R}\right) \\
(n=1,2),
\end{gathered}
$$

where

$$
\begin{aligned}
& d^{R}=\left(\frac{2}{\pi R \cos \theta}\right)^{1 / 2}\left(1-8 \tan ^{2} \theta\right)^{-1 / 4}, \\
& d_{k}^{S}=\exp \left(-\gamma Q_{k}\left|z_{0}\right|\right), \\
& \varphi_{k}=\gamma Q_{k}^{1 / 2} R\left(\cos \theta-q_{k} \sin \theta \tan \theta\right)+(-1)^{k+1} \pi / 4,
\end{aligned}
$$

and the definitions of $R, \theta, q_{k}$, and $Q_{k}$ can be found in the Appendix.

\section{INTERFACIAL WAVES BETWEEN AN UPPER VISCOUS AND A LOWER INVISCID FLUID}

\section{A. Mathematical formulation}

In this section, the upper fluid is assumed to be viscous while the lower fluid inviscid. The fundamental singularity in the viscous Oseen flow is an Oseenlet, which represents a point force acting on the fluid. For the upper fluid, the dimensionless governing equations are

$$
\nabla \cdot \mathbf{u}_{1 n}=0,
$$

$$
\frac{\partial \mathbf{u}_{1 n}}{\partial x}=-\nabla P_{1 n}+\varepsilon_{1} \nabla^{2} \mathbf{u}_{1 n}+\Delta_{1 n} \mathbf{F} \delta\left(\mathbf{x}-\mathbf{x}_{0}\right),
$$

where $\mathbf{u}_{1 n}=\left(u_{1 n}, v_{1 n}, w_{1 n}\right)$ is the disturbed velocity field due to a singularity immersed in fluid $n, P_{1 n}$ is the corresponding hydrodynamic pressure, and $\varepsilon_{1}=\left(\mu_{1} g\right) /\left(\rho_{1} U^{3}\right)$, where $\mu_{1}$ is the dynamic viscosity of the upper fluid. $\mathbf{F}=(-F, 0,0)$ is the dimensionless forcelet acting on the upper fluid. The dimensionless parameter $\varepsilon_{1}$ can be regarded as the reciprocal of the Reynolds number with respect to the characteristic length. For the lower fluid, the dimensionless governing equation is

$$
\nabla^{2} \Phi_{2 n}=\Delta_{2 n} M \delta\left(\mathbf{x}-\mathbf{x}_{0}\right) .
$$

For small-amplitude waves, we impose the linearized boundary conditions on the undisturbed interface $(z=0)$. The kinematic conditions on the interface are given by

$$
\frac{\partial \zeta_{n}}{\partial x}=w_{1 n}=\frac{\partial \Phi_{2 n}}{\partial z}
$$

where $\zeta_{n}$ is the dimensionless elevation of the interface between the upper viscous and lower inviscid fluid. The dynamic conditions on the interface are given by

$$
\begin{aligned}
& \varepsilon_{1}\left(\frac{\partial u_{1 n}}{\partial z}+\frac{\partial w_{1 n}}{\partial x}\right)=0, \\
& \varepsilon_{1}\left(\frac{\partial v_{1 n}}{\partial z}+\frac{\partial w_{1 n}}{\partial y}\right)=0,
\end{aligned}
$$




$$
\sigma\left(P_{1 n}-\zeta_{n}-2 \varepsilon_{1} \frac{\partial w_{1 n}}{\partial z}\right)=-\frac{\partial \Phi_{2 n}}{\partial x}-\zeta_{n}
$$

Equation (25) represents the vanishing of shearing stress in the $x$ direction while Eq. (26) in the $y$ direction. Equation (27) represents the balance of the normal stresses.

\section{B. Integral solutions}

For the upper viscous fluid the disturbed flow is regarded as the sum of an unbounded singular Oseen flow, which represents the effect of the singular forcelet, and a bounded regular Oseen flow, which represents the influence of the interface. Thus, we write

$$
\left[\mathbf{u}_{1 n}, P_{1 n}\right]=\left[\mathbf{u}_{1 n}^{R}(\mathbf{x}), P_{1 n}^{R}(\mathbf{x})\right]+\Delta_{1 n}\left[\mathbf{u}_{1 n}^{S}\left(\mathbf{x} ; \mathbf{x}_{0}\right), P_{1 n}^{S}\left(\mathbf{x} ; \mathbf{x}_{0}\right)\right] .
$$

Furthermore, the continuous vector $\mathbf{u}_{1 n}^{R}$ is taken as the sum of an irrotational and a solenoidal vector, $\mathbf{u}_{1 n}^{R}=\boldsymbol{\nabla} \Phi_{1 n}^{R}+\mathbf{V}_{1 n}^{T}$, where $\Phi_{1 n}^{R}$ is a harmonic function and $\mathbf{V}_{1 n}^{T}$ is a solenoidal vector. Thus,

$$
\begin{aligned}
& \nabla^{2} \Phi_{1 n}^{R}=0, \\
& \nabla \cdot \mathbf{V}_{1 n}^{T}=0, \\
& \frac{\partial \mathbf{V}_{1 n}^{T}}{\partial x}=\varepsilon_{1} \nabla^{2} \mathbf{V}_{1 n}^{T}, \\
& P_{1 n}^{R}=-\frac{\partial \Phi_{1 n}^{R}}{\partial x} .
\end{aligned}
$$

For the velocity potential of the lower inviscid fluid, the same splitting as in Eq. (6) will be applied. Therefore, the boundary conditions can be expressed in terms of $\Phi_{1 n}^{R}, \mathbf{V}_{1 n}^{T}$, $\Phi_{2 n}^{R}, \mathbf{u}_{11}^{S}$, and $\Phi_{22}^{S}$ on the undisturbed interface $(z=0)$,

$$
\begin{aligned}
& \left(\frac{\partial \Phi_{1 n}^{R}}{\partial z}+w_{1 n}^{T}\right)-\frac{\partial \Phi_{2 n}^{R}}{\partial z}=-\Delta_{1 n} w_{11}^{S}+\Delta_{2 n} \frac{\partial \Phi_{22}^{S}}{\partial z}, \\
& \frac{\partial \zeta_{n}}{\partial x}-\left(\frac{\partial \Phi_{1 n}^{R}}{\partial z}+w_{1 n}^{T}\right)=\Delta_{1 n} w_{11}^{S}, \\
& 2 \frac{\partial^{2} \Phi_{1 n}^{R}}{\partial x \partial z}+\frac{\partial u_{1 n}^{T}}{\partial z}+\frac{\partial w_{1 n}^{T}}{\partial x}=-\Delta_{1 n}\left(\frac{\partial u_{11}^{S}}{\partial z}+\frac{\partial w_{11}^{S}}{\partial x}\right), \\
& 2 \frac{\partial^{2} \Phi_{1 n}^{R}}{\partial y \partial z}+\frac{\partial v_{1 n}^{T}}{\partial z}+\frac{\partial w_{1 n}^{T}}{\partial y}=-\Delta_{1 n}\left(\frac{\partial v_{11}^{S}}{\partial z}+\frac{\partial w_{11}^{S}}{\partial y}\right), \\
& (\sigma-1) \zeta_{n}+\sigma \frac{\partial \Phi_{1 n}^{R}}{\partial x}+2 \sigma \varepsilon_{1}\left(\frac{\partial^{2} \Phi_{1 n}^{R}}{\partial z^{2}}+\frac{\partial w_{1 n}^{T}}{\partial z}\right)-\frac{\partial \Phi_{2 n}^{R}}{\partial x} \\
& \quad=\Delta_{1 n} \sigma\left(P_{11}^{S}-2 \varepsilon_{1} \frac{\partial w_{11}^{S}}{\partial z}\right)+\Delta_{2 n} \frac{\partial \Phi_{22}^{S}}{\partial x} .
\end{aligned}
$$

By invoking the Fourier transform and the Cauchy residue theorem, the integral-form fundamental solutions of Eqs. (21) and (22) due to a forcelet $\mathbf{F}=(-F, 0,0)$ located at $\mathbf{x}_{0}$ can be obtained as $^{25}$

$$
\begin{aligned}
\mathbf{u}_{1 n}^{S}\left(\mathbf{x} ; \mathbf{x}_{0}\right)= & \frac{1}{8 \pi^{2}} \mathbf{F} \cdot\left(\nabla \nabla-\mathbf{I} \nabla^{2}\right) \int_{-\infty}^{+\infty} \int_{-\infty}^{+\infty} d \alpha d \beta \frac{\exp (i f)}{i \alpha} \\
& \times\left[\frac{1}{K} \exp \left(-K\left|z-z_{0}\right|\right)\right. \\
& \left.-\frac{1}{B_{1}} \exp \left(-B_{1}\left|z-z_{0}\right|\right)\right] \\
P_{1 n}^{S}\left(\mathbf{x} ; \mathbf{x}_{0}\right)= & -\frac{1}{8 \pi^{2}}(\mathbf{F} \cdot \nabla) \int_{-\infty}^{+\infty} \int_{-\infty}^{+\infty} d \alpha d \beta \frac{1}{K} \\
& \times \exp \left(i f-K\left|z-z_{0}\right|\right)
\end{aligned}
$$

where $B_{1}=\sqrt{i \alpha / \varepsilon_{1}+K^{2}}$.

In order to obtain the formal solution for interfacial waves, a joint integral transform is introduced as

$$
\begin{aligned}
& {\left[\widetilde{\zeta}_{n}, \widetilde{\Phi}_{1 n}^{R}, \widetilde{\mathbf{V}}_{1 n}^{T}, \widetilde{\Phi}_{2 n}^{R}\right]} \\
& \quad=\int_{-\infty}^{+\infty} \int_{-\infty}^{+\infty} d \alpha d \beta \exp (-i f) \\
& \quad \times\left[\zeta_{n}, \Phi_{1 n}^{R} \exp (K z), V_{1 n}^{T} \exp \left(B_{1} z\right), \Phi_{2 n}^{R} \exp (-K z)\right] .
\end{aligned}
$$

By taking transform (40) over the continuity equation (30) and Eqs. (33)-(37), we obtain a system of linear equations for $\left[\widetilde{\zeta}_{n}, \widetilde{\Phi}_{1 n}^{R}, \tilde{\mathbf{V}}_{1 n}^{T}, \widetilde{\Phi}_{2 n}^{R}\right]$, which can readily be solved. Upon some mathematical manipulation, the integral-form solutions for the wave profiles on the interface can be written as

$\zeta_{n}=\frac{\lambda}{4 \pi^{2}} \int_{-\infty}^{+\infty} \int_{-\infty}^{+\infty} \frac{A_{1 n}}{D_{1}} \exp ($ if $) d \alpha d \beta \quad(n=1,2)$,

where

$A_{11}=-\sigma F\left[\left(i \alpha+2 \varepsilon_{1} K^{2}\right) \exp \left(-K z_{0}\right)-2 \varepsilon_{1} K B_{1} \exp \left(-B_{1} z_{0}\right)\right]$,

$A_{12}=i \alpha M \exp \left(K z_{0}\right)$

$D_{1}=\gamma K-\alpha^{2}+4 i \varepsilon_{1} \alpha K^{2} \tau+4 \varepsilon_{1}^{2} K^{3}\left(K-B_{1}\right) \tau$,

and $\tau=\sigma /(1+\sigma)=\rho_{1} /\left(\rho_{1}+\rho_{2}\right) . D_{1}$ in Eq. (44) may be regarded as the dispersion function for the interfacial waves between an upper viscous and a lower inviscid fluid.

\section{Asymptotic representations}

Next, the asymptotic representations of Eq. (41) in the far field for large Reynolds numbers, which are of principal physical interest, will be derived by means of Lighthill's two-stage scheme. The reciprocal of the Reynolds number, $\varepsilon_{1}$, will be used as the perturbation parameter throughout the asymptotic analysis.

For small $\varepsilon_{1}$, the dispersion equation has two zeros with respect to $\alpha$,

$$
\alpha_{j}=(-1)^{j+1} a_{0}+i \varepsilon_{1} a_{1}+O\left(\varepsilon_{1}^{3 / 2}\right) \quad(j=1,2),
$$

where $a_{1}(\beta)=4 \tau a_{0}^{6}\left(2 a_{0}^{2}-\gamma^{2}\right)^{-1}$, and $a_{0}$ is given by Eq. (A1) (see the Appendix). 
The approximation of the phase function near the saddle points is similar to Lurye's Eq. (52) (Ref. 7) and Lu's Eq. (45) (Ref. 9). The analysis involving the first term on the right-hand side of Eq. (45) follows that in the Appendix, while the analysis involving $\varepsilon_{1}$ follows those given by Lurye, ${ }^{7}$ Chan and Chwang, ${ }^{8}$ and Lu. ${ }^{9}$ The procedure will not be reproduced here. The asymptotic representations for the far-field interfacial waves can be written as

$$
\begin{aligned}
& \zeta_{1}=\sum_{l=0}^{2} \zeta_{1 l}+o\left(\frac{1}{R}\right), \\
& \zeta_{2}=\lambda \gamma^{1 / 2} M d^{R} \sum_{k=1}^{2} d_{k}^{S} d_{1 k}^{V} Q_{k}^{3 / 4} \cos \varphi_{k}+o\left(\frac{1}{R}\right),
\end{aligned}
$$

where

$$
\begin{aligned}
\zeta_{10}= & -\tau \gamma^{1 / 2} F d^{R} \sum_{k=1}^{2} d_{k}^{S} d_{1 k}^{V} Q_{k}^{3 / 4} \cos \varphi_{k}, \\
\zeta_{11}= & -2 \varepsilon_{1} \tau \gamma^{3 / 2} F d^{R} \sum_{k=1}^{2} d_{k}^{S} d_{1 k}^{V} Q_{k}^{9 / 4} \sin \varphi_{k}, \\
\zeta_{12}= & 2 \varepsilon_{1}^{1 / 2} \tau \gamma F d^{R} \sum_{k=1}^{2} d_{1 k}^{B} d_{1 k}^{V} Q_{k}^{3 / 2} \\
& \times \sin \left(\varphi_{k}-\frac{\gamma^{1 / 2} Q_{k}^{1 / 4}\left|z_{0}\right|}{\sqrt{2 \varepsilon_{1}}}+\frac{\pi}{4}\right), \\
d_{1 k}^{V}= & \exp \left(-4 \varepsilon_{1} \tau \gamma^{4} q_{k}^{-1} Q_{k}^{3} R \cos \theta\right), \\
d_{1 k}^{B}= & \exp \left(-\frac{\gamma^{1 / 2} Q_{k}^{1 / 4}\left|z_{0}\right|}{\sqrt{2 \varepsilon_{1}}}\right) .
\end{aligned}
$$

\section{INTERFACIAL WAVES BETWEEN AN UPPER INVISCID AND A LOWER VISCOUS FLUID}

In this section, the upper fluid is assumed to be inviscid while the lower fluid to be viscous. The governing equations and boundary conditions can be obtained by switching subscripts 1 and 2 in Eqs. (21)-(26) and by replacing $\zeta_{n}$ by $\eta_{n}$ and Eq. (27) by

$$
-\sigma\left(\frac{\partial \Phi_{1 n}}{\partial x}+\eta_{n}\right)=P_{2 n}-\eta_{n}-2 \varepsilon_{2} \frac{\partial w_{2 n}}{\partial z},
$$

where $\eta_{n}$ is the elevation of the interface between the upper inviscid and lower viscous fluid, $\varepsilon_{2}=\left(\mu_{2} g\right) /\left(\rho_{2} U^{3}\right), \mu_{2}$ the dynamic viscosity of the lower fluid, and $w_{2 n}$ the velocity component in the $z$ direction.

The procedure to obtain the integral-form solutions for the wave profiles on the interface follows that in Sec. III and will not be reproduced here. Upon some mathematical manipulation, we have

$$
\eta_{n}=\frac{\lambda}{4 \pi^{2}} \int_{-\infty}^{+\infty} \int_{-\infty}^{+\infty} \frac{A_{2 n}}{D_{2}} \exp (\text { if }) d \alpha d \beta \quad(n=1,2),
$$

where

$$
\begin{aligned}
& A_{21}=-i \alpha \sigma M \exp \left(-K z_{0}\right), \\
& A_{22}=F\left[\left(i \alpha+2 \varepsilon_{2} K^{2}\right) \exp \left(K z_{0}\right)-2 \varepsilon_{2} K B_{2} \exp \left(B_{2} z_{0}\right)\right], \\
& D_{2}=\gamma K-\alpha^{2}+4 i \varepsilon_{2} \alpha K^{2} \lambda+4 \varepsilon_{2}^{2} K^{3}\left(K-B_{2}\right) \lambda, \\
& B_{2}=\sqrt{i \alpha / \varepsilon_{2}+K^{2}} .
\end{aligned}
$$

The application of Lighthill's two-stage scheme to Eq. (54) yields the asymptotic representations for the far-field interfacial waves as

$$
\begin{aligned}
& \eta_{1}=-\tau \gamma^{1 / 2} M d^{R} \sum_{k=1}^{2} d_{k}^{S} d_{2 k}^{V} Q_{k}^{3 / 4} \cos \varphi_{k}+o\left(\frac{1}{R}\right), \\
& \eta_{2}=\sum_{l=0}^{2} \zeta_{2 l}+o\left(\frac{1}{R}\right),
\end{aligned}
$$

where

$$
\begin{aligned}
\eta_{20}= & \lambda \gamma^{1 / 2} F d^{R} \sum_{k=1}^{2} d_{k}^{S} d_{2 k}^{V} Q_{k}^{3 / 4} \cos \varphi_{k}, \\
\eta_{21}= & 2 \varepsilon_{2} \lambda \gamma^{3 / 2} F d^{R} \sum_{k=1}^{2} d_{k}^{S} d_{2 k}^{V} Q_{k}^{9 / 4} \sin \varphi_{k}, \\
\eta_{22}= & -2 \varepsilon_{2}^{1 / 2} \lambda \gamma F d^{R} \sum_{k=1}^{2} d_{2 k}^{B} d_{2 k}^{V} Q_{k}^{3 / 2} \\
& \times \sin \left(\varphi_{k}-\frac{\gamma^{1 / 2} Q_{k}^{1 / 4}\left|z_{0}\right|}{\sqrt{2 \varepsilon_{2}}}+\frac{\pi}{4}\right), \\
d_{2 k}^{V}= & \exp \left(-4 \varepsilon_{2} \lambda \gamma^{4} q_{k}^{-1} Q_{k}^{3} R \cos \theta\right), \\
d_{2 k}^{B}= & \exp \left(-\frac{\gamma^{1 / 2} Q_{k}^{1 / 4}\left|z_{0}\right|}{\sqrt{2 \varepsilon_{2}}}\right) .
\end{aligned}
$$

\section{DISCUSSION}

First, the effect of density ratio on the interfacial wave profile is analyzed. It can be seen from Eq. (17) that for a fixed $\left|z_{0}\right|, \xi_{1}=-\sigma \xi_{2}$. There is a phase shift of $\pi$ between them, as shown in Fig. 1. When the effect of the upper fluid disappears (i.e., $\sigma=0, \gamma=\lambda=1$, and $n=2$ ), Eq. (16) simply reduces to Crapper's Eq. (15) (Ref. 26). for the classical dispersion relation of ship waves in a single inviscid fluid of infinite depth, while Eq. (17) does so to Wehausen and Laitone's Eq. (13.42) (Ref. 1) for the inviscid ship waves. Equation (57) simply reduces to Cumberbatch's Eq. (25) (Ref. 3) for the complex dispersion relation of ship waves in a single viscous fluid while Eq. (60) does so to Lu's Eq. (47) (Ref. 9) for viscous free-surface waves due to a horizontal Oseenlet. 


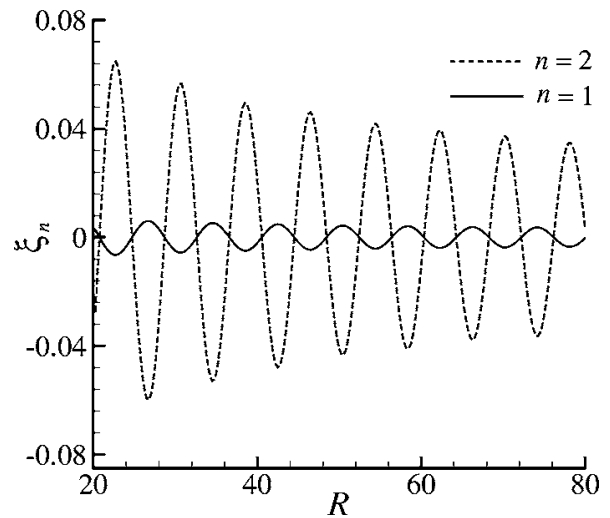

FIG. 1. Interfacial waves due to a singularity moving in the upper or lower inviscid fluid with $M=1, \sigma=0.1, \quad \theta=\frac{1}{2} \tan ^{-1} \sqrt{1 / 8}, z_{0}=+1$, and $z_{0}=-1$, respectively.

Lurye's Eqs. (69) and (70) (Ref. 7) and Chan and Chwang's Eqs. (69) and (70) (Ref. 8) for $0<\theta<\tan ^{-1} \sqrt{1 / 8}$ are also recovered by Eq. (60). Mathematical proof was provided by $\mathrm{Lu}^{27}$ for the equivalency between Lurye's ${ }^{7}$ and Lu's ${ }^{9}$ analytical representations for viscous ship waves in the region 0 $<\theta<\tan ^{-1} \sqrt{1 / 8}$. The solutions for a single fluid, inviscid or viscous, are recovered by the results presented here for a two-fluid system.

The interfacial inviscid wave patterns may be characterized by constant phase lines, which are described by parametric equations

$$
\begin{aligned}
& x=\frac{2 l \pi+(-1)^{k} \pi / 4}{\gamma Q_{k}^{1 / 2}\left(1-q_{k} \tan ^{2} \theta\right)}, \\
& y=\frac{\left[2 l \pi+(-1)^{k} \pi / 4\right] \tan \theta}{\gamma Q_{k}^{1 / 2}\left(1-q_{k} \tan ^{2} \theta\right)},
\end{aligned}
$$

where $\theta$ is the parameter and $k=1,2, l=0,1,2, \ldots$. It is clear that the wave patterns resemble the classical Kelvin ship wave pattern in a single fluid. A remarkable conclusion can be made about the wavelengths of interfacial waves, which are defined by

$$
L=2 \pi\left[\gamma Q_{k}^{1 / 2}\left(\cos \theta-q_{k} \sin \theta \tan \theta\right)\right]^{-1} \quad(k=1,2) .
$$

The effect of parameter $\sigma$ on the wavelength is shown in Fig. 2. Close examination of Eqs. (66) and (67) and Fig. 2 shows that the expression involving $q_{1}$ corresponds to the so-called transverse waves, while the expression involving $q_{2}$ corresponds to the so-called diverging waves. It can be seen from Eq. (68) and Fig. 2 that as $\theta$ tends to $\tan ^{-1} \sqrt{1 / 8}$, the wavelength of all steady waves tends to $4 \sqrt{3} \pi /(3 \gamma)$. As $\theta$ tends to zero, the wavelength of transverse waves tends to $2 \pi / \gamma$ while that of diverging waves tends to zero. Figure 3 shows the interfacial waves between two inviscid fluids with a singularity immersed in the lower one. It is found that the wavelength is elongated in comparison with that of free-surface waves on a single fluid $(\sigma=0)$. As the density ratio $\sigma$ becomes larger, the wavelength becomes longer. In the limit as $\sigma$ approaches 1 , the wavelengths go to infinity.

To study the effect of density ratio on the interfacial inviscid wave amplitude, a wave envelope is defined as

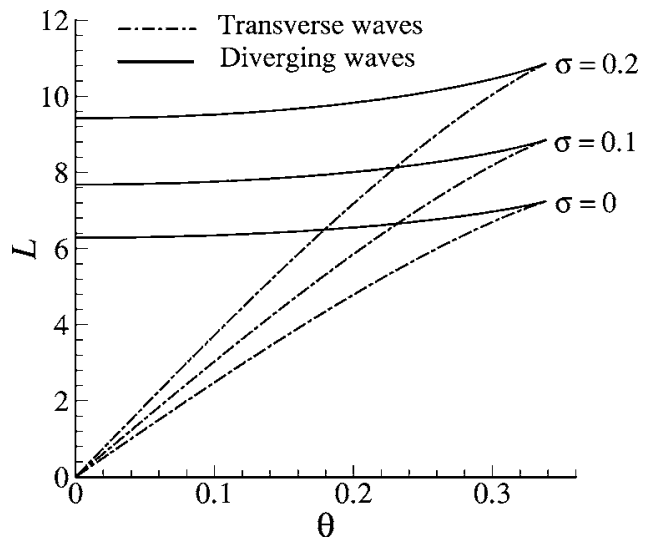

FIG. 2. The effect of density ratio on the wavelength.

$$
E_{n k}\left(M, \sigma,\left|z_{0}\right| ; R, \theta\right)=\sigma^{2-n} \lambda \gamma^{1 / 2} M d^{R} d_{k}^{S} Q_{k}^{3 / 4} \quad(n, k=1,2) .
$$

The stationary points of wave envelope $E_{n k}$ with respect to $\sigma$, denoted by $\sigma_{n k}$, can be determined by

$$
\frac{d E_{n k}}{d \sigma}=0 .
$$

Since $0<\sigma<1$ and $Q_{k} \geqslant 1$, the solutions of Eq. (70) in reality are

$$
\begin{aligned}
& \sigma_{1 k}=\frac{-1+2 Q_{k}\left|z_{0}\right|+\sqrt{9+4 Q_{k}\left|z_{0}\right|+4 Q_{k}^{2} z_{0}^{2}}}{4\left(1+Q_{k}\left|z_{0}\right|\right)} \quad(k=1,2), \\
& \sigma_{2 k}=\frac{1}{2}\left(1+2 Q_{k}\left|z_{0}\right|-\sqrt{9-4 Q_{k}\left|z_{0}\right|+4 Q_{k}^{2} z_{0}^{2}}\right) \quad(k=1,2),
\end{aligned}
$$

at which the transverse/diverging wave envelopes might reach their extrema when $M,\left|z_{0}\right|$, and $\theta$ are fixed. It is noted that $\sigma_{1 k}$ and $\sigma_{2 k}$ are independent of $R$. Figures 4-6 show the transverse, diverging, and resultant wave components, respectively, versus the density ratio for $M=1, z_{0}=-1$, and $\theta$ $=\frac{1}{2} \tan ^{-1} \sqrt{1 / 8}$. By Eq. (72), we have the corresponding stationary points for the transverse and diverging wave enve-

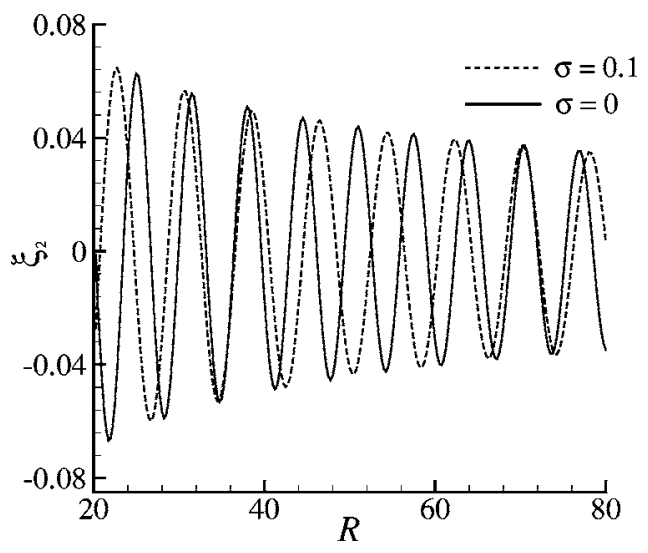

FIG. 3. The effect of density ratio on wave profiles with $M=1, \theta$ $=\frac{1}{2} \tan ^{-1} \sqrt{1 / 8}$, and $z_{0}=-1$. 


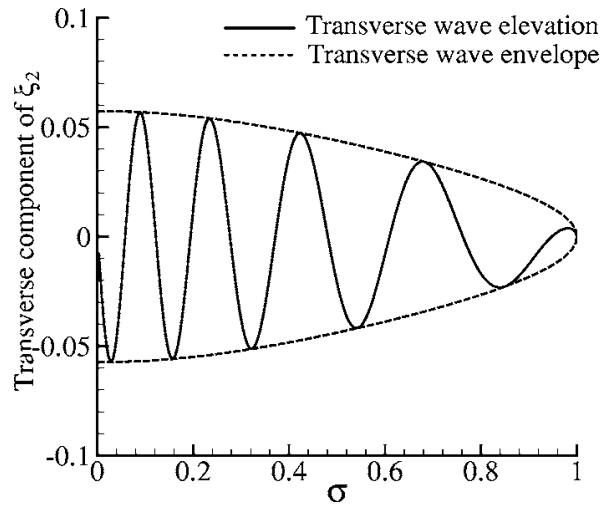

FIG. 4. The transverse wave component vs the density ratio for $M=1, z_{0}$ $=-1, R=30$, and $\theta=\frac{1}{2} \tan ^{-1} \sqrt{1 / 8}$.

lopes shown in Figs 4 and 5, i.e., $\sigma_{21} \approx 0.0220137$ and $\sigma_{22} \approx 0.875334$, respectively. It is well known that the freesurface wave system due to a submerged source moving in a single inviscid fluid $(\sigma=0)$ primarily consists of transverse waves $^{2}$ because the diverging waves are heavily damped by a submergence decay factor $\exp \left(Q_{k} z_{0}\right)$ with $z_{0}<0$, especially near the path of moving source $(\theta=0)$. However, the contribution from the transverse and diverging components to the interfacial wave profiles presented in Eq. (17) depends on the density ratio. Figures 4 and 5 show that as the density ratio increases from zero, the transverse component first rises to the extremum at $\sigma_{21}$ and then decreases, while the diverging component first increases gradually from zero to its extremum at $\sigma_{22}$ and then drops rapidly. Figure 6 shows that the maximum resultant wave height appears near $\sigma_{22}$. In the limit as $\sigma$ approaches 1, as shown in Figs. 4-6, the wave height drops rapidly to zero, which indicates that the singularity moving in a homogeneous fluid does not produce wave motions in the far field.

Next, the effect of viscosity on the wave profile is considered. When the viscosity of the upper fluid tends to zero $\left(\varepsilon_{1} \rightarrow 0\right)$, Eq. (41) simply reduces to Eq. (14) if $F=M$ while Eqs. (46) and (47) reduces to Eq. (17). When the viscosity of the lower fluid tends to zero $\left(\varepsilon_{2} \rightarrow 0\right)$, Eq. (54) simply reduces to Eq. (14) if $F=M$ while Eqs. (59) and (60) simply reduce to Eq. (17). It is observed that the governing equa-

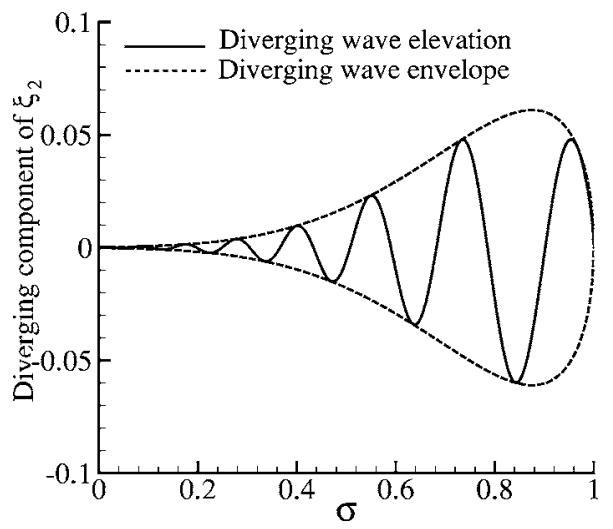

FIG. 5. The diverging wave component vs the density ratio for $M=1, z_{0}$ $=-1, R=30$, and $\theta=\frac{1}{2} \tan ^{-1} \sqrt{1 / 8}$.

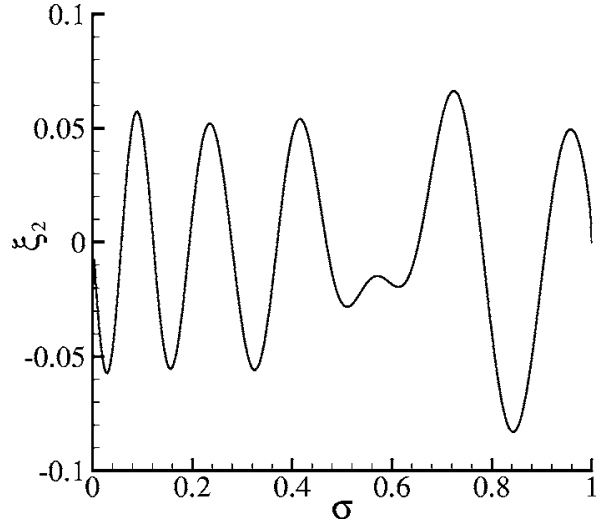

FIG. 6. The resultant wave amplitude vs the density ratio for $M=1, z_{0}$ $=-1, R=30$, and $\theta=\frac{1}{2} \tan ^{-1} \sqrt{1 / 8}$.

tions and boundary conditions for the two-inviscid-fluid system cannot be obtained by simply dropping the terms involving viscosity for the system with a viscous and an inviscid fluid. However, as far as the wave motion at the interface is concerned, the solutions for ship waves in an inviscid fluid are recovered from those for a viscous fluid as the viscosity tends to zero. As for the system consisting of a viscous and an inviscid fluid, it can be seen from Eqs. (47) and (59) that the interfacial waves due to the moving source immersed in the inviscid one are damped by viscous decay factors. A viscous wake, however, will be generated when the singularity moves in the viscous fluid. The additive terms $\zeta_{11}, \zeta_{12}$, $\eta_{21}$, and $\eta_{22}$ represent the effect of viscous wake on the interfacial wave amplitude and vanish as the viscosity tends to zero. Figure 7 shows the effect of viscosity for different physical configurations. It is noted that as $\sigma$ increases from zero to $1, \tau$ increases from zero to $1 / 2$ while $\lambda$ decreases from 1 to 1/2. By a comparison between Eq. (51) and Eq. (64) and from Fig. 7, it can be concluded that for a small $\sigma$, $\eta_{n}$ is more heavily damped than $\zeta_{n},(n=1,2)$.

It should be noted that all the solutions obtained here are valid for $|\theta|<\tan ^{-1} \sqrt{1 / 8}$. The analytical solution for the interfacial waves at $|\theta|=\tan ^{-1} \sqrt{1 / 8}$ can be calculated by using Ursell's method ${ }^{28}$ for the inviscid system and Cumberbatch's

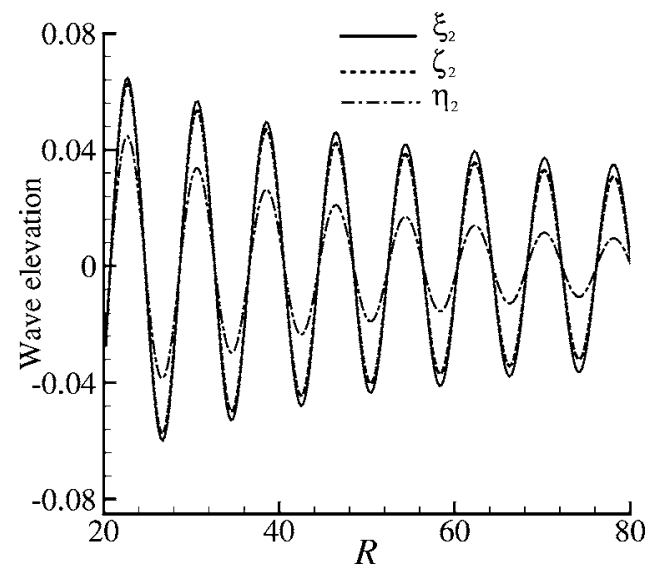

FIG. 7. The wave elevation for $M=F=1, z_{0}=-1, \sigma=0.1, \varepsilon_{1}=\varepsilon_{2}=0.01$, and $\theta=\frac{1}{2} \tan ^{-1} \sqrt{1 / 8}$. 
$\operatorname{method}^{3}$ for the viscous system. For $|\theta|>\tan ^{-1} \sqrt{1 / 8}$, there are no waves. The wave region of validity for the interfacial waves considered here is the same as that for Kelvin ship waves on the free surface of a single fluid of infinite depth.

In the framework of the linear potential theory for ship motion in a single inviscid fluid, Chen and $\mathrm{Wu}^{29}$ revealed that the highly oscillatory and singular properties of the Green's function occur when the field point approaches the track of the source point located at the free surface. One can see from Eq. (17) that the interfacial inviscid waves will tend to infinity when both $z_{0}$ and $\theta$ approach zero. It has been demonstrated by Cumberbatch, ${ }^{3}$ Chan and Chwang, ${ }^{8}$ and $\mathrm{Lu}^{9}$ that the highly oscillatory and singular behavior can be removed by the inclusion of viscosity in the mathematical formulation of ship waves. Likewise, one can verify that Eqs. (46), (47), (59), and (60) predict finite wave amplitudes due to the presence of viscous decay factors. Recently, $\mathrm{Chen}^{30}$ proposed another approach to eliminate the nonphysical behavior by introducing the surface tension for ship waves in an inviscid fluid. The combined effect of viscosity and surface tension on singularity induced waves remains a mathematical task and will be studied in the future.

\section{CONCLUSIONS}

By means of Lighthill's two-stage scheme, the asymptotic solutions for the interfacial waves due to a fundamental singularity moving above or beneath the interface are obtained for three systems: one with two inviscid fluids, one with an upper viscous and a lower inviscid fluid, and one with an upper inviscid and a lower viscous fluid. The physical interpretations for interfacial waves are explicitly given. The previous works $^{7-9}$ for a single viscous fluid are extended. It is felt that the mathematical models and methodology presented here can be extended to the system consisting of two semi-infinite viscous fluids. However, a more complicated mathematical analysis will be involved.

It is found that the wavelength of interfacial waves is elongated in comparison with that of free-surface waves in a single fluid. For small density ratio, the contribution from transverse waves to the wave elevation is predominant and the contribution from diverging waves is insignificant. As the density ratio increases, the transverse waves decrease while the diverging waves become significant. When the density difference between the two fluids is small, the maximum wave amplitude occurs. A theoretical prediction for such a density ratio is analytically presented.

\section{ACKNOWLEDGMENTS}

This research was sponsored by the Hong Kong Research Grants Council under Grant No. HKU 7076/02E and the State Key Laboratory of Ocean Engineering, China, under Grant No. 0502. The work of D.Q.L. was partially supported by the Shanghai Leading Academic Discipline Project under Project No. Y0103.

\section{APPENDIX: ASYMPTOTIC SCHEME}

In accordance with Lighthill's theory, the asymptotic behavior of the wave integral in Eq. (14) is predominated by the contribution from poles of the integrand. It is easily seen that $D_{0}$ has two simple zeros with respect to $\alpha$,

$\alpha_{j}=(-1)^{j+1} a_{0}(\beta)=(-1)^{j+1} \sqrt{\gamma^{2}+\gamma \sqrt{\gamma^{2}+4 \beta^{2}}} \quad(j=1,2)$.

It should be noted that the poles lie on the real axis in the Fourier plane. In order to derive a correct physical solution for the wave propagation, it has long been customary to introduce a "fictitious viscosity" to move the poles off the real axis. The solution for the original problem can be obtained as the "fictitious viscosity" tends to zero, as stated by Yeung and Nguyen. ${ }^{19}$

We introduce the cylindrical coordinates $(R, \theta)$ on the horizontal $(x, y)$ plane by

$$
x=R \cos \theta, y=R \sin \theta .
$$

Using the Cauchy residue theorem, the leading terms which contribute significantly to the far-field wave profiles can be written as

$$
\begin{aligned}
\xi_{n}= & \frac{(-1)^{n+1} \sigma^{2-n} \lambda M}{2 \pi} \sum_{j=1}^{2} \int_{-\infty}^{+\infty}\left\{\frac{a_{0}^{2}}{\gamma^{2}-2 a_{0}^{2}}\right. \\
& \left.\times \exp \left[i R f_{j}+(-1)^{n} \gamma^{-1} a_{0}^{2} z_{0}\right]+o\left(\frac{1}{R}\right)\right\} \mathrm{d} \beta,
\end{aligned}
$$

where

$$
f_{j}(\beta)=\alpha_{j} \cos \theta+\beta \sin \theta .
$$

The integrals in Eq. (A2) can further be approximated by the method of stationary phase. The dominant contribution to the far-field wave profiles stems from the stationary points of the phase equations, which can be determined by

$$
\frac{\partial f_{j}(\beta)}{\partial \beta}=\frac{\partial}{\partial \beta}\left[(-1)^{j+1} a_{0} \cos \theta+\beta \sin \theta\right]=0 .
$$

To solve Eq. (A4), a change of variable is introduced as

$$
\beta(b)=(-1)^{j} \frac{b}{\gamma} \sqrt{\gamma^{2}+b^{2}} .
$$

Thus

$$
a_{0}(b)=\sqrt{\gamma^{2}+b^{2}} .
$$

The substitution of Eqs. (A5) and (A6) into Eq. (A4) yields

$$
(-1)^{j+1} \frac{\partial}{\partial b}\left[\left(\cos \theta-\frac{b}{\gamma} \sin \theta\right) \sqrt{\gamma^{2}+b^{2}}\right] \frac{\partial b}{\partial \beta}=0 .
$$

Taking a straightforward mathematical manipulation on Eq. (A7), we have the identity

$$
\gamma^{2}+b^{2}=\frac{1}{2}\left(\frac{\gamma b}{\tan \theta}+\gamma^{2}\right)
$$

and the solutions of Eq. (A7) 


$$
b_{k}=\gamma q_{k} \tan \theta,
$$

where

$$
q_{k}=2\left[1+(-1)^{k+1} \sqrt{1-8 \tan ^{2} \theta}\right]^{-1} \quad(k=1,2) .
$$

The substitution of Eq. (A9) into Eqs. (A8), (A5), and (A1) yields the formal expressions for the stationary points $\left(\alpha_{j k}, \beta_{j k}\right)$ as

$$
\begin{aligned}
& \alpha_{j k}(\theta)=(-1)^{j+1} a_{0}\left(b_{k}\right)=(-1)^{j+1} \gamma Q_{k}^{1 / 2}, \\
& \beta_{j k}(\theta)=(-1)^{j} \gamma q_{k} Q_{k}^{1 / 2} \tan \theta,
\end{aligned}
$$

where

$$
Q_{k}=\left(q_{k}+1\right) / 2 \quad(j, k=1,2) .
$$

Near the stationary points, we can put

$$
\begin{aligned}
\xi_{n}= & \frac{(-1)^{n+1} \sigma^{2-n} \lambda M}{2 \pi} \sum_{j=1}^{2} \sum_{k=1}^{2} \frac{a_{0 k}^{2}}{\gamma^{2}-2 a_{0 k}^{2}} \\
& \times \exp \left[i R f_{j k}+(-1)^{n} \gamma^{-1} a_{0 k}^{2} z_{0}\right] \\
& \times \int_{-\infty}^{+\infty} \exp \left[\frac{R}{2} \frac{\partial^{2} f_{j}\left(\beta_{j k}\right)}{\partial \beta^{2}}\left(\beta-\beta_{j k}\right)^{2}\right] \mathrm{d} \beta+o\left(\frac{1}{R}\right),
\end{aligned}
$$

where

$$
f_{j k}=\alpha_{j k} \cos \theta+\beta_{j k} \sin \theta .
$$

By a standard stationary-phase approximation, we have Eq. (17).

${ }^{1}$ J. V. Wehausen and E. V. Laitone, "Surface waves," in Encyclopedia of Physics IX: Fluid Dynamics III, edited by S. Flügge (Springer, Berlin, 1960), pp. 446-815.

${ }^{2}$ Y. K. Chung and J. S. Lim, "A review of the Kelvin ship wave pattern," J. Ship Res. 35, 191 (1991).

${ }^{3}$ E. Cumberbatch, "Effects of viscosity on ship waves," J. Fluid Mech. 23, $471(1965)$

${ }^{4}$ J. R. Lurye, "Interaction of free surface waves with viscous wakes," Phys. Fluids 11, 261 (1968).

${ }^{5}$ M. J. Lighthill, "Studies on magneto-hydrodynamic waves and other anisotropic wave motions," Philos. Trans. R. Soc. London 252, 397 (1960).

${ }^{6} \mathrm{~S}$. L. Wen, "An extension of Lighthill's result on asymptotic evaluation of multiple Fourier integrals," Int. J. Eng. Sci. 7, 53 (1969).

${ }^{7}$ J. R. Lurye, "Wave height and wave resistance in the presence of a viscous wake,” Phys. Fluids 16, 750 (1973).
${ }^{8}$ A. T. Chan and A. T. Chwang, "Interaction of laminar far wake with a free surface," Phys. Fluids 8, 421 (1996).

${ }^{9}$ D. Q. Lu, "Interaction of viscous wakes with a free surface," Appl. Math. Mech. Engl. Ed. 25, 647 (2004).

${ }^{10}$ A. T. Chan and A. T. Chwang, "Ship waves on a viscous fluid of finite depth," Phys. Fluids 9, 940 (1997).

${ }^{11}$ M. J. Liu and M. D. Tao, "Transient ship waves on an incompressible fluid of infinite depth," Phys. Fluids 13, 3610 (2001).

${ }^{12}$ D. Q. Lu and A. T. Chwang, "Unsteady free-surface waves due to a submerged body moving in a viscous fluid," Phys. Rev. E 71, 066303 (2005).

${ }^{13}$ M. J. Liu and M. D. Tao, "Velocity field in ship waves on the viscous fluid," Appl. Math. Mech. Engl. Ed. 23, 1221 (2002).

${ }^{14} \mathrm{D}$. Q. Lu, "Free-surface waves and far wakes generated by a floating body in a viscous fluid," J. Hydrodynam. Ser. B 15, 10 (2003).

${ }^{15}$ J. H. Milgram, "Theory of radar backscatter from short waves generated by ships, with application to radar (SAR) imagery," J. Ship Res. 32, 54 (1988).

${ }^{16}$ A. M. Reed and J. H. Milgram, "Ship wakes and their radar images," Annu. Rev. Fluid Mech. 34, 469 (2002).

${ }^{17}$ Y. S. He, C. J. Lu, and X. N. Chen, "Analytical solutions of singularities moving with an arbitrary path when two fluids are present," Appl. Math. Mech. Engl. Ed. 12, 131 (1991).

${ }^{18}$ D. Palaniappan, "General slow viscous flows in a two-fluid system," Acta Mech. 193, 1 (2000).

${ }^{19}$ R. W. Yeung and T. C. Nguyen, "Waves generated by a moving source in a two-layer ocean of finite depth," J. Eng. Math. 35, 85 (1999).

${ }^{20}$ W. Gang, D. Q. Lu, and S. Q. Dai, "Waves induced by a submerged moving dipole in a two-layer fluid of finite depth," Acta Mech. Sin. 21, 24 (2005).

${ }^{21}$ D. Q. Lu and A. T. Chwang, in Recent Advances in Fluid Mechanics: Proceedings of the Fourth International Conference on Fluid Mechanics, Dalian, China, 20-23 July 2004, edited by F. G. Zhuang and J. C. Li (Tsinghua University Press and Springer, Beijing, 2004), pp. 292-295.

${ }^{22}$ A. T. Chwang and T. Y. T. Wu, "Hydromechanics of low-Reynoldsnumber flow. Part 2. Singularity method for Stokes flows," J. Fluid Mech. 67, 787 (1975).

${ }^{23}$ D. G. Duffy, Transform Methods for Solving Partial Differential Equations (CRC, Boca Raton, FL, 1994), p. 117.

${ }^{24}$ F. Noblesse and X. B. Chen, "Decomposition of free-surface effects into wave and near-field components," Ship Technology Res. 42, 167 (1995).

${ }^{25}$ D. Q. Lu and A. T. Chwang, in Proceedings of the 17th National Conference on Hydrodynamics and the Sixth National Congress on Hydrodynamics, Hong Kong, China, edited by L. D. Zhou, W. W. Shao, C. J. Lu, and W. H. Hui (China Ocean Press, Beijing, 2003), pp. 283-291.

${ }^{26}$ G. D. Crapper, "Surface waves generated by a traveling pressure point," Proc. R. Soc. London, Ser. A 282, 547 (1964).

${ }^{27} \mathrm{D}$. Q. Lu, "A note on the complex dispersion relation for steady viscous ship waves," J. Hydrodynam. Ser. B 17, 22 (2005).

${ }^{28}$ F. Ursell, "On the Kelvin's ship-wave pattern," J. Fluid Mech. 8, 418 (1960).

${ }^{29} \mathrm{X}$. B. Chen and G. X. Wu, "On singular and highly-oscillatory properties of the Green function for ship motions," J. Fluid Mech. 445, 77 (2001).

${ }^{30} \mathrm{X} . \mathrm{B}$. Chen, in Proceedings of the 17th International Workshop on Water Waves and Floating Bodies, edited by R. C. T. Rainey and S. F. Lee (Peterhouse, Cambridge, UK, 2002), pp. 25-28. 